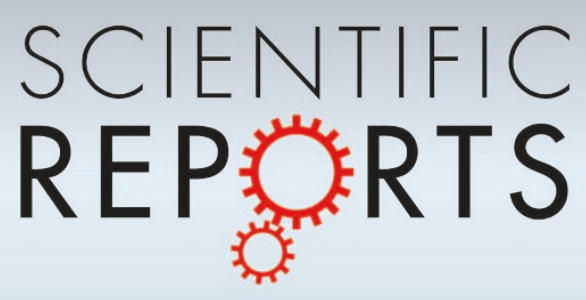

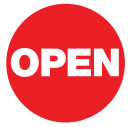

SUBJECT AREAS:

SENSORS AND

BIOSENSORS

ELECTRONIC PROPERTIES AND

MATERIALS

TWO-DIMENSIONAL MATERIALS

ELECTRONIC DEVICES

Received

21 November 2012

Accepted

17 December 2012

Published

4 February 2013

Correspondence and requests for materials should be addressed to Z.Y.Z. (zyzhang@pku. edu.cn) or L.M.P. (Impeng@pku.edu.cn)

\section{Batch-fabricated high-performance graphene Hall elements}

\author{
Huilong $X u^{1}$, Zhiyong Zhang ${ }^{1}$, Runbo Shi ${ }^{1}$, Honggang Liv' ${ }^{2}$, Zhenxing Wang' ${ }^{1}$, Sheng Wang' \\ \& Lian-Mao Peng'
} ${ }^{1}$ Key Laboratory for the Physics and Chemistry of Nanodevices and Department of Electronics, Peking University, Beijing 100871,
China, ${ }^{2}$ Microwave Devices and Integrated Circuits Department, Institute of Microelectronics, Chinese Academy of Sciences, Beijing
100029, China.

Hall elements are by far the most widely used magnetic sensor. In general, the higher the mobility and the thinner the active region of the semiconductor used, the better the Hall device. While most common magnetic field sensors are Si-based Hall sensors, devices made from III-V compounds tend to favor over that based on Si. However these devices are more expensive and difficult to manufacture than $\mathrm{Si}$, and hard to be integrated with signal-processing circuits for extending function and enforcing performance. In this article we show that graphene is intrinsically an ideal material for Hall elements which may harness the remarkable properties of graphene, i.e. extremely high carrier mobility and atomically thin active body, to create ideal magnetic sensors with high sensitivity, excellent linearity and remarkable thermal stability.
M agnetic sensors are devices capable of sensing a magnetic field and extracting information from it. Among them, Hall elements are by far the most widely used. More than two billion Hall elements are manufactured annually, and used in such fields ranging from computers to sewing machines, automobiles to aircraft, machine tools to medical equipment, and industrial applications to scientific detecting ${ }^{1-5}$. Hall devices exploit the galvanomagnetic effects due to the Lorentz force on charge carriers in a semiconductor, and transform a magnetic signal into an electric signal. In general, the higher the mobility of the semiconductor used, and the thinner the active region, the better the Hall device ${ }^{5}$. While most common magnetic field sensors are Sibased Hall sensors, devices made from III-V compounds, and particularly narrow-gap, high-mobility compounds such as InSb, tend to favor over that based on $\mathrm{Si}^{6-9}$, and ultra-high sensitivity Hall devices have also been realized based on two-dimensional-electron-gas (2DEG) which combines ultra-high carrier mobility with thin activebody ${ }^{6,10,11}$. However these devices are more expensive and difficult to manufacture than $\mathrm{Si}$, and hard to be integrated with signal-processing circuits for extending function and enforcing performance ${ }^{6-11}$.

Graphene has been considered as a promising material for electronics applications in recent years, largely due to its extremely thin body and ultra-high carrier mobility ${ }^{12}$. Extensive investigations have been carried out on graphene field-effect transistors (G-FETs) which hold promise for radio-frequency applications ${ }^{12,13}$. However GFETs currently perform no better than conventional transistors based on silicon or III-V compound semiconductors $^{12}$. This may be largely attributed to the fact that the graphene device fabrication technology is still immature and falls far behind the conventional semiconductor technology, and this completely offsets the material advantages of graphene. In principle a more predictable electronics application of graphene should focus on a kind of simple device which only requires a very simple fabrication process while takes the full advantages of the remarkable physical properties of graphene. Compared with FET and other electrical devices ${ }^{12,14,15}$. Hall element is much simpler in device structure and is thus much easier to fabricate. The performance of a Hall element is mainly dependent on the electric properties of the material, most importantly on carrier mobility and concentration ${ }^{1-6}$, rather than on fabrication technology. Since graphene is born with extremely high room temperature carrier mobility and atomically thin body, it is an ideal material for building highperformance Hall elements. In addition, since FET based integrated circuits (ICs) can also be fabricated on graphene ${ }^{12-13}$, the function of Hall elements can be further enhanced in the future by combining them with those ICs on the same chip. It is thus nature to conclude that graphene could be the ideal material for building highperformance Hall elements and ICs with lower cost and better performance than conventional Hall devices, leading perhaps to the first field of mass electronic applications for graphene.

Although Hall effects including quantum Hall effects of graphene were intensively studied from the viewpoints of physical interests ${ }^{16}$, the performance of graphene Hall structure as a device aiming for sensor applications was 
hardly investigated. Recently a graphene magnetometer based on magnetoresistance effect was demonstrated, with potential applications in such fields as scanning probe magnetometry and magnetic storage $^{17,18}$. Micro-sized Hall devices were also fabricated using graphene and important parameters including sensitivity and resolution were studied ${ }^{19,20}$. However, limited by the quality of the graphene used and not optimized device geometry and contact resistance, a low field resolution of $2.5 \mu \mathrm{T} / \sqrt{\mathrm{Hz}}$ was reported ${ }^{20}$ which lags far behind that achieved by low-noise silicon-on-insulator Hall device of about $400 \mathrm{nT} / \sqrt{\mathrm{Hz}}$. In principle high quality graphene should be an ideal conductive channel for building Hall elements. Here we show, by utilizing the methods for growing and transferring high quality and large area graphene which we developed recently ${ }^{21}$, graphene Hall elements (GHEs) with higher performances than conventional Hall elements in all important aspects, including higher sensitivity and resolution, higher linearity, lower driving voltage, wider temperature range and better thermal stability can be realized and batch-fabricated. In addition, key performances of GHEs, in particular sensitivity, are controllable during the fabricating process of the devices according to the needs of desired applications. In this letter we show that graphene is intrinsically an ideal material for Hall elements which may harness the remarkable properties of graphene, i.e. extremely high carrier mobility and atomically thin active body, to create ideal magnetic sensors with high sensitivity of more than $1200 \mathrm{~V} / \mathrm{AT}$, excellent linearity of less than $\pm 2 \%$ for magnetic field of up to $2 \mathrm{~T}$ and remarkable thermal stability from $2 \mathrm{~K}$ to $400 \mathrm{~K}$. In addition, graphene Hall elements can be batch-fabricated with low cost and readily integrated with integrated circuits (ICs) for signalamplifying and processing.

\section{Results}

A macro GHE with a channel length and width larger than $100 \mu \mathrm{m}$ was firstly fabricated on a large area graphene on $\mathrm{Si} / \mathrm{SiO}_{2}$ substrate. The macro GHE was then used to construct a graphene Gauss meter as a high sensitivity magnetic detector (see supplementary information for detailed description of the Gauss meter). After calibrated by a high sensitivity commercial Gauss meter, the graphene Gauss meter was used to map a two-dimensional non-uniform magnetic field. The output Hall voltages of the GHE were recorded at various positions of the magnetic field, and the magnetic field strength at corresponding position was compared with that measured by using the commercial Gauss meter. Quantitative analysis reveals that the Hall voltage of the GHE is linearly dependent on the magnetic field strength as measured by the commercial Gauss meter with very small fluctuations (Fig. 1a), and the GHE can thus be used as a Gauss meter after calibration through the measured linear relation to yield a twodimensional map of magnetic field distribution as shown in Fig. 1b. This map is very similar to that mapped using the commercial Gauss meter in which a high sensitivity InSb Hall element is used as the magnetic detector (see Fig. S1f in supplementary information). The small difference between the two maps may come from the positioning differences between the two measurements which were carried out manually and the performance fluctuation of the GHE where the graphene channel was exposed directly to air during the measurement process.

Comparing to macro Hall elements, micro Hall elements with channel shorter than $10 \mu \mathrm{m}$ afford higher density and higher spatial resolution $^{1,4}$. Micro GHEs were fabricated on a $\mathrm{Si} / \mathrm{SiO}_{2}$ substrate with a size of about $2 \mathrm{~cm} \times 2 \mathrm{~cm}$ (Fig. $2 \mathrm{a}$ ), which was covered with a transferred high-quality graphene ${ }^{21}$. Over 400 GHEs were batch-fabricated on the $2 \mathrm{~cm} \times 2 \mathrm{~cm}$ substrate with approximately $50 \%$ yield. While not yet wafer-scaled, the procedure used here may in principle be scaled up to yield wafer-scale graphene material grown and devices fabrication process with higher yield than $50 \%$.

Figure $2 \mathrm{~b}$ presents a scanning electron microscopic (SEM) image showing a typical cross-shaped micro GHE, which is a four-terminal (a)

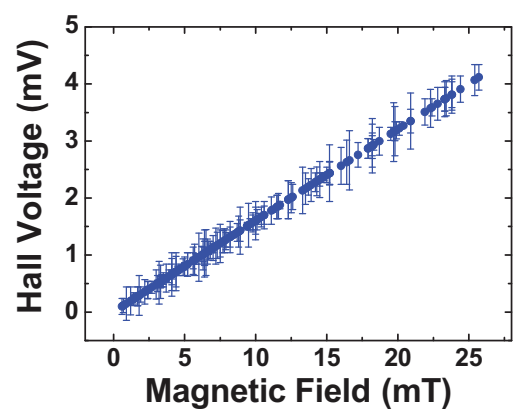

(b)

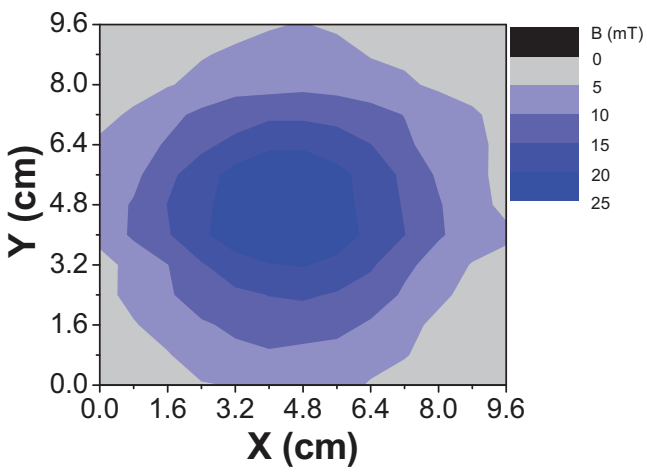

Figure $1 \mid$ Macro GHE as the detector of a Gauss meter. (a) Output Hall voltages of a GHE sampled at different points of an external magnetic field as a function of field strength measured by a commercial high sensitivity Gauss meter. (b) Two-dimensional map of the external magnetic field measured using the calibrated GHE.

device with two pairs of electrodes contacting four terminals of the graphene Hall cross. One pair electrodes $\left(C_{1}\right.$ and $\left.C_{2}\right)$ are designed for current or voltage supply, and the other pair $\left(\mathrm{C}_{3}\right.$ and $\left.\mathrm{C}_{4}\right)$ are for Hall voltage measurement. When an external magnetic field $\mathrm{B}$ is applied normal to the graphene plane, with the graphene channel being biased either with a constant current or voltage supply over $C_{1}$ and $\mathrm{C}_{2}$, a Hall voltage is developed over $\mathrm{C}_{3}$ and $\mathrm{C}_{4}$ which depends linearly on the strength of the magnetic field as a result of the Hall effect, i.e. $V_{\mathrm{H}}=S_{\mathrm{A}} B$, where $S_{\mathrm{A}}$ is the absolute sensitivity of the Hall element ${ }^{5,6}$. In principle GHEs can be used as magnetometers if the detailed electrical characteristics of the devices are known.

A Hall element can be used either in constant-current-supply or current mode, or in constant-voltage-supply or voltage mode. The Hall voltage generated in current mode can be written as ${ }^{1,6}$

$$
V_{\mathrm{H}}=S_{\mathrm{I}} I B \text {, }
$$

and in voltage mode

$$
V_{\mathrm{H}}=S_{\mathrm{V}} V B \text {, }
$$

where $S_{\mathrm{I}}=\left.\frac{1}{\mathrm{I}} \frac{\partial V_{\mathrm{H}}}{\partial B}\right|_{\mathrm{I}=\text { Const }}$ and $S_{\mathrm{V}}=\left.\frac{1}{\mathrm{~V}} \frac{\partial V_{\mathrm{H}}}{\partial B}\right|_{\mathrm{V}=\text { Const }}$ are current-related sensitivity and voltage-related sensitivity respectively, and I or $\mathrm{V}$ is the constant current or voltage supplied. The sensitivity of a sensor represents its ability to respond to a signal to be measured (here a magnetic field), and is one of the key performance parameters of the Hall element ${ }^{1-11}$.

We first consider the current mode which is the most widely used mode for conventional Hall elements, since this mode may effectively avoid the degradation of device linearity induced by the magnetoresistance effect. When a GHE is measured in current mode, its output Hall voltage as a function of the magnetic field is shown in Fig. 2c. This figure shows clearly that the output Hall voltage increases linearly with increasing supply current and magnetic field ranging from 
(a)

(b)

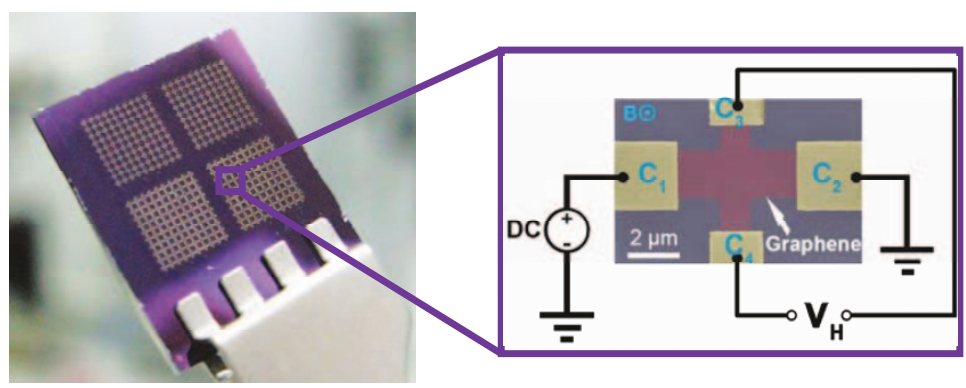

(c)

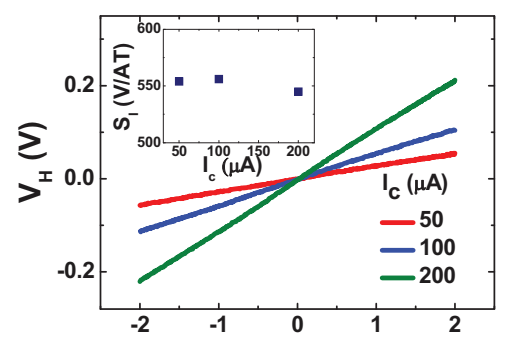

(d)

(e)

Field (T)
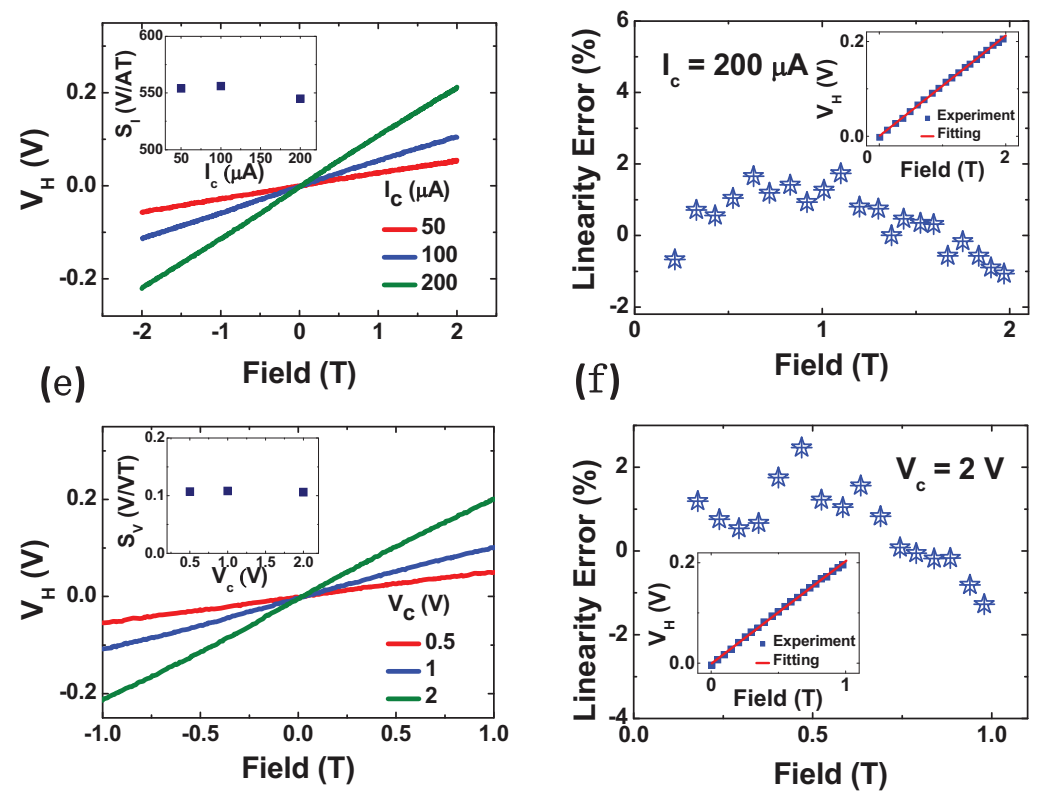

Figure 2 Batch-fabricated micro GHEs and their room temperature characteristics. (a) Optical image showing $\mathrm{GHEs}$ arrays on a Si/SiO $\mathrm{S}_{2}$ chip. (b) SEM image showing one of the GHEs of (a) and related Hall testing circuitry. (c) Hall voltage versus magnetic field measured in current mode. Inset: current-related sensitivity. (d) Linearity error of the GHE working in current mode and in a magnetic field ranging from 0 to 2 T. Inset: a typical linear fitting of the Hall voltage versus magnetic field. (e) Hall voltage versus magnetic field in voltage mode. Inset: voltage-related sensitivity. (f) Linearity error of the GHE working in voltage mode and in a magnetic field ranging from 0 to 1 T. Inset: a typical linear fitting of the Hall voltage versus magnetic field.

$-2 \mathrm{~T}$ to $2 \mathrm{~T}$, suggesting a very large measurement range for magnetic field. The slopes of the $V_{\mathrm{H}}$-B curves give the absolute sensitivity $S_{\mathrm{A}}$ of the GHE, which increases also linearly with bias current. Normalizing the absolute sensitivity $S_{\mathrm{A}}$ with the bias current, the current-related sensitivity $S_{\mathrm{I}}$ of the GHE is obtained and shown in the inset of Fig. 2c. The current-related sensitivity of the micro-GHE exceeds $550 \mathrm{~V} / \mathrm{AT}$ at room temperature. As a comparison, for a typical conventional Hall devices ${ }^{1}$, these values are around $100 \mathrm{~V} /$ AT for silicon Hall plates fabricated by the standard bulk CMOS IC technology ${ }^{5}$, and typically $200 \mathrm{~V} / \mathrm{AT}$ for GaAs (for example the standard GaAs Hall element provided by Asahi Kasei Corporation has a sensitivity $200 \mathrm{~V} / \mathrm{AT}$ ), but may approach $700 \mathrm{~V} / \mathrm{AT}$ for 2DEGbased Hall devices ${ }^{1}$.

Linearity, an important performance parameter of Hall devices, determines the accuracy of measured result and is quantitatively described by a linearity error defined as ${ }^{7}$

$$
\alpha=\left(V_{\mathrm{H}}-V_{\mathrm{H}}^{0}\right) / V_{\mathrm{H}}^{0},
$$

where $V_{\mathrm{H}}{ }^{0}$ is the best linear fitted value to the measured Hall voltages $V_{\mathrm{H}}$ at a given magnetic field. The data shown in Fig. 2d are obtained by linear fitting $V_{\mathrm{H}}$ - B curves (e.g. those shown in Fig. 2c) for various magnetic field strengths ranging from 0 to $2 \mathrm{~T}$. The linearity error lies within $\pm 2 \%$, suggesting that the GHE is a highly linear Hall element in current mode over a large magnetic field range.
The voltage mode is another operation mode of Hall devices. But few conventional Hall elements can directly work in this mode with high linearity over a wide range of magnetic fields as a result of the magnetoresistance effect. This is particularly so for the physical magnetoresistance effect, which results from the mobility spread of the charge carriers around the Fermi energy and usually scales up with magnetic field via a square relationship ${ }^{5,7}$. The output Hall voltage as a function of the magnetic field in voltage mode is given in Fig. 2e, showing clearly that the output Hall voltage increases linearly with increasing magnetic field and also with the supply voltage. The slope of the $V_{\mathrm{H}}$-B curve gives the absolute sensitivity $S_{\mathrm{A}}$ of the GHE, and voltage-related sensitivity $S_{\mathrm{V}}$ of the GHE is obtained to be about $0.1 \mathrm{~V} / \mathrm{VT}$ through normalizing $S_{\mathrm{A}}$ with bias voltage, as shown in the inset of Fig. 2e.

For real applications the linearity is perhaps the most concerned parameter for Hall elements working in voltage mode, since the linearity is usually degraded seriously due to the magnetoresistance effect in conventional Hall elements. While in GHEs, $V_{\mathrm{H}}$ is hardly affected by the effect, which is a result of the absence of the physical magnetoresistance in graphene in the Coulomb scattering range (see supplementary information). The inset of Fig. $2 \mathrm{f}$ shows that the Hall voltage $V_{\mathrm{H}}$ may be linearly fitted with B excellently well for a wide range of magnetic field from 0 to $1 \mathrm{~T}$, and typically the linearity error is within $\pm 2 \%$. This small error should be compared with the typical 
value of about $10 \%$ for 2 DEGs Hall elements at magnetic field less than $0.1 \mathrm{~T}^{10}$. GHEs thus afford excellent linearity over a wide magnetic field range in voltage mode, in which the use of Hall elements are indeed more convenient and economic than in current mode. Operating in voltage mode, GHEs are not only simpler to power, but also show better stability than in current mode. As a typical example, Fig. $2 S$ of supplementary information shows back gate voltage dependence of both the voltage-related and current-related sensitivities. It is obvious that the voltage-related sensitivity shows a much weaker dependence on the back gate (or on environment) than the current-related sensitivity. This is because the carrier density is a much more sensitive parameter to the gate voltage or environment than the carrier mobility. It should be pointed out that our GHE is still far from being optimized both on device geometry and fabricating process, which leaves a large space for further improvements. A simple derivation shows that the sensitivity of a Hall element is strongly dependent on carrier density or mobility, and to a good approximation current and voltage related sensitivities of a Hall element can be written as ${ }^{1,6}$

$$
\begin{gathered}
S_{I}=\frac{1}{q n_{s}}, \\
S_{V}=\frac{\mu W}{L},
\end{gathered}
$$

where $q$ is the elemental charge, $W$ and $L$ are the width and length, and $n_{s}$ and $\mu$ are the sheet carrier density and carrier mobility of the graphene channel respectively. Therefore the sensitivity of the GHE can be improved by decreasing ns or increasing carrier mobility $\mu$, and also by optimizing the geometry of the device.

(a)
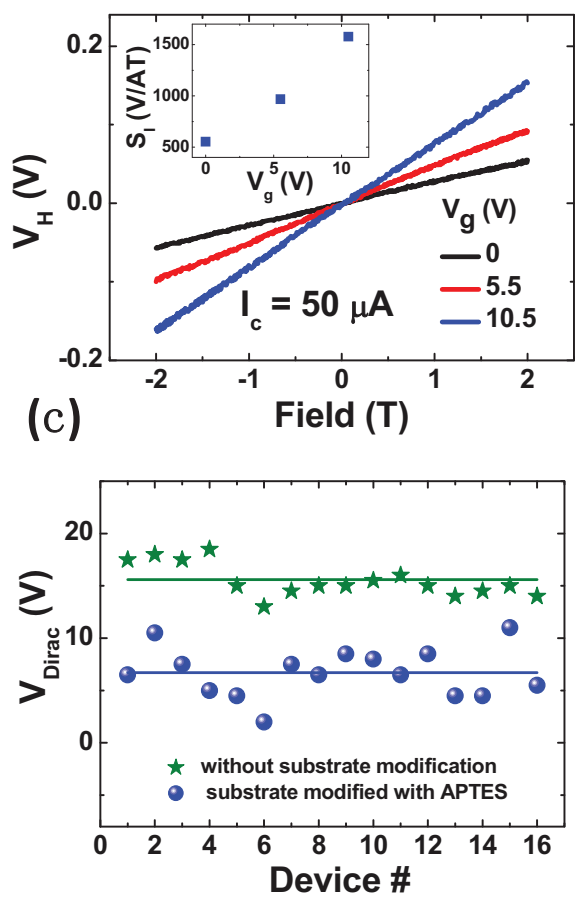

For a typical graphene channel, carrier density $n_{s}$ can in principle be controlled by applying a voltage $V_{\mathrm{g}}$ on the back gate ${ }^{12}$, i.e. the $\mathrm{Si}$ substrate here, then the current sensitivity $S_{\text {I }}$ of the GHE can be adjusted through gate voltage as shown in Fig. 3a. Since the Dirac voltage of the GHE is about $13 \mathrm{~V}$ (Fig. 3b), the carrier density (or device current) may be significantly reduced by applying a positive gate voltage smaller than $13 \mathrm{~V}$ on the back gate. The inset of Fig. 3a shows that $\mathrm{S}_{\mathrm{I}}$ increases from $550 \mathrm{~V} / \mathrm{AT}$ at $V_{\mathrm{g}}=0 \mathrm{~V}$ to about $1500 \mathrm{~V} /$ AT at $V_{\mathrm{g}}=10.5 \mathrm{~V}$, while corresponding carrier density decreases from $1 \times 10^{12} / \mathrm{cm}^{2}$ to about $4 \times 10^{11} / \mathrm{cm}^{2}$. Although gate voltage can be easily used to adjust the sensitivity of the GHE, the use of an additional large voltage on the back gate would bring great inconvenience to device fabrication and applications ${ }^{19}$. Alternatively we may emulate the back gate effect by surface or substrate modification. It is by now well established that the Dirac voltage of a graphene Hall device can be adjusted through modifying the surface of $\mathrm{Si} / \mathrm{SiO}_{2}$ substrate with 3aminopropyltriethoxysilane (APTES) before graphene was transferred onto the surface ${ }^{22}$. Figure $3 \mathrm{~b}$ compares the transfer characteristics of a GHE with and without the additional modification treatment of the surface. This figure shows clearly that the GHE with APTES modification exhibits a smaller Dirac voltage and larger transconductance than that without surface modification. Thirty-two Hall elements were fabricated and measured, and half of the devices were fabricated on functionalized $\mathrm{Si} / \mathrm{SiO}_{2}$ substrate. Statistical results (Fig. 3c) show that the Dirac voltage can be reduced systematically from $15.5 \mathrm{~V}$ to $6.7 \mathrm{~V}$ by the additional modification process (all transfer curves of the two groups of devices are shown in Fig. S3 in supplementary information). The current sensitivity of the GHE is improved up to 1200 V/AT for the GHE fabricated on functionalized surface (Fig. 3d) which is much larger than that of most commercial Hall elements. (b)
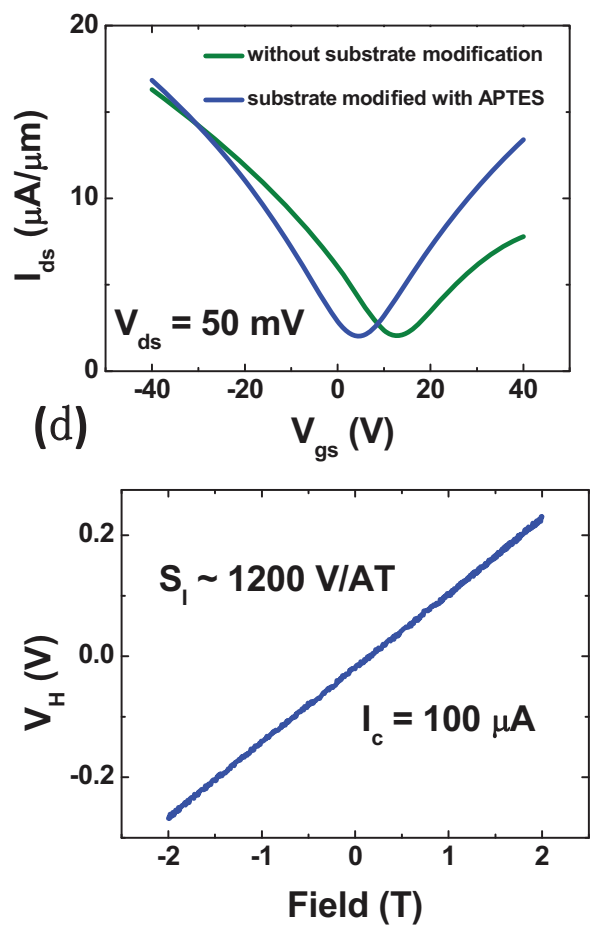

Figure 3 Controlled Dirac point voltage and Hall elements sensitivity. (a) Hall voltage versus magnetic field in current mode and at varying back gate voltage. Inset: current-related sensitivity as a function of the back gate voltage. (b) Transfer characteristics of two typical GHEs at a bias of $50 \mathrm{mV}$. The olive curve represents the GHE being fabricated through the normal process, and the blue curve represents that being fabricated on a substrate which was modified by APTES before graphene was transferred. (c) Statistical comparison of Dirac point voltages of GHEs with or without substrate modification. (d) Hall voltage versus magnetic field of a typical GHE with APTES modification in current mode without applying back gate voltage. 
(a)

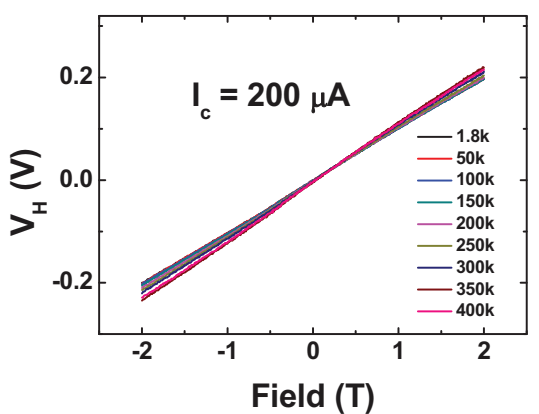

(c)

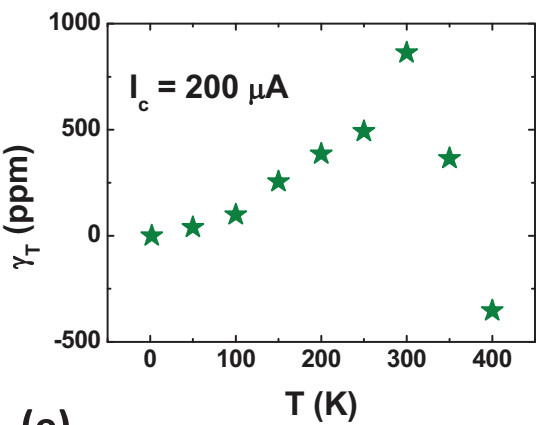

(e)

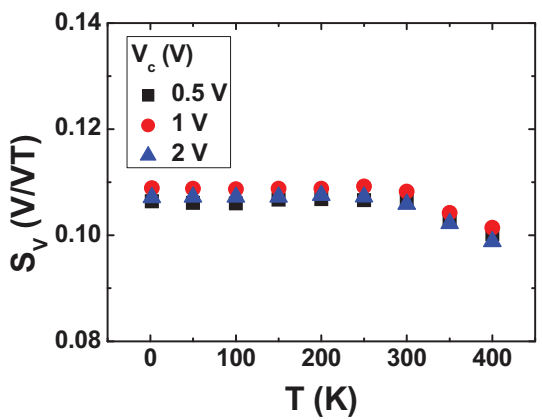

(b)

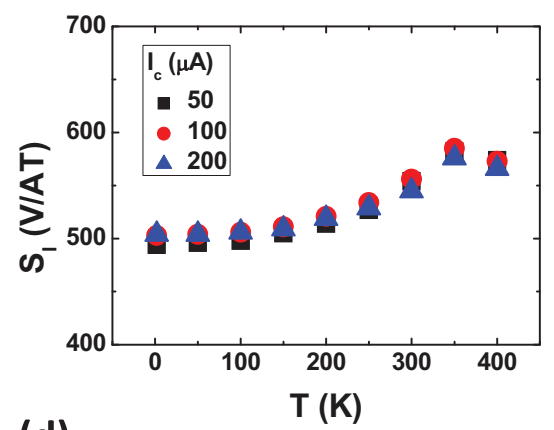

(d)

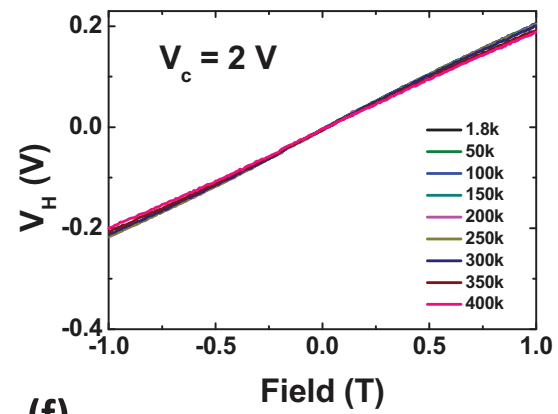

(f)

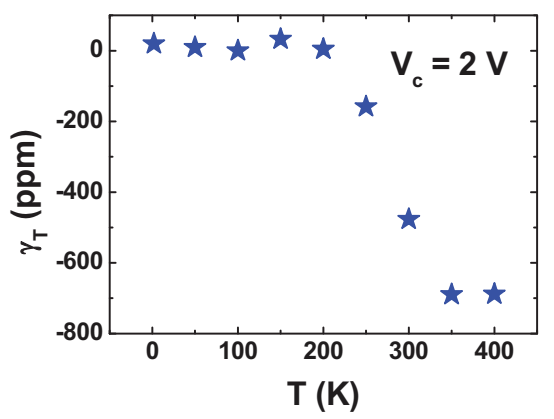

Figure 4 Temperature performance of GHE. (a) Hall voltage versus magnetic field in current mode (with a drive current of $200 \mu \mathrm{A}$ ) and over a wide temperature range from $1.8 \mathrm{~K}$ to $400 \mathrm{~K}$. (b) The corresponding current-related sensitivities at various temperatures and (c) the related temperature coefficients. (d) Hall voltage versus magnetic field working in voltage mode (with a drive voltage of $2 \mathrm{~V}$ ) and over a wide temperature range from $1.8 \mathrm{~K}$ to $400 \mathrm{~K}$. (e) The corresponding voltage-related sensitivities at various temperatures and (f) the related temperature coefficients.

To further demonstrate the potential of GHEs in real applications, we tested nine APTES-treated micro GHEs in atmosphere conditions. The performance of the nine tested devices is similar to that tested in vacuum conditions (see the detailed in supplementary information). The current-related sensitivity at room temperature ranges from $645 \mathrm{~V} / \mathrm{AT}$ to $1180 \mathrm{~V} / \mathrm{AT}$, with an average value of $850 \mathrm{~V} / \mathrm{AT}$ [Figure S4(b)]. The voltage-related sensitivity ranges from $0.2 \mathrm{~V} / \mathrm{VT}$ to $0.35 \mathrm{~V} / \mathrm{VT}$, with an average value of about $0.3 \mathrm{~V} / \mathrm{VT}$ [Figure S4(d)]. The Hall mobility extracted based on equation (5) is found to distribute around $5000 \mathrm{~cm}^{2} / \mathrm{V} \cdot \mathrm{s}$ [Figure S4(d)], suggesting that our CVD graphene samples are of high quality. It should be noted that the tests were performed in air within 1 hour. Longer exposure time of the devices in air may result in contamination of graphene channel by possible organic or water molecules, and as a result degradation of the devices performance may occur. Further passivation technique is needed so as to deliver stable and repeatable performance of GHEs.

Graphene devices are also intrinsically low noise devices ${ }^{23}$. The combination of high sensitivity and low noise of graphene devices ${ }^{23}$ may in principle lead to very high resolution, i.e. minimum detectable field $\mathrm{B}_{\mathrm{min}}$, and in our case this is less than $800 \mathrm{nT} / \sqrt{\mathrm{Hz}}$ at operation frequency of $3 \mathrm{kHz}$ (see e.g. Figure S5 of supplementary information). The resolution achieved here presents a significant improvement on that achieved previously ${ }^{19,20}$ which is larger than $2.5 \mu \mathrm{T} / \sqrt{\mathrm{Hz}}$ even at higher operation frequency and lower temperature. Increasing the operation frequency up to $4.5 \mathrm{MHz}, \mathrm{B}_{\min }$ can be improved to about $50 \mathrm{nT} / \sqrt{\mathrm{Hz}}$. Normalized by the device width, the resolution of the GHE is about $100 \mathrm{pT} \cdot \mathrm{mm} / \mathrm{Hz}^{1 / 2}$ which is much smaller than that of most conventional Hall elements ${ }^{1}$. In principle the sensitivity and resolution of the GHE can be further improved by optimizing substrate and growth conditions ${ }^{24,25}$. It is well known that for graphene it is possible to lower $n_{s}$ to about $1.2 \times 10^{11} \mathrm{~cm}^{-2}$ and to improve $\mu$ up to about $100,000 \mathrm{~cm}^{2} / \mathrm{V} \cdot \mathrm{s} .{ }^{24,25}$ Then $S_{\text {I }}$ of a GHE can in principle be improved up to $5000 \mathrm{~V} / \mathrm{AT}$, which is significantly larger than that of any conventional Hall elements, and a $S_{\mathrm{V}}$ of up to about $7 \mathrm{~V} / \mathrm{VT}$ (the maximum effective width-to-length ratio $\sim 0.7$ has been taken into account ${ }^{5}$, which limits the maximum voltage related sensitivity as $S_{\mathrm{Vmax}} \sim 0.7 \mu$ ) which also compares favorably with the best Hall devices based on InP, InAs or even $2 \mathrm{DEG}^{1-11}$. The ultra-high normalized resolution $\left(\mathrm{B}_{\min } \cdot \mathrm{W}\right)$ of up to $4 \mathrm{pT} \cdot \mathrm{mm} / \mathrm{Hz}^{1 / 2}$ can also be expected in the GHE at room temperature (detailed estimating process is described in the Methods section), which points to the potential detection of an extremely weak field of picotesla.

In addition to high sensitivity and linearity, excellent stability over a wide temperature range is also crucial for high performance Hall 
elements, and will significantly extend the application fields of Hall elements $^{26}$. Figure $4 \mathrm{a}$ shows the output Hall voltage as a function of the magnetic field at a range of temperatures from $1.8 \mathrm{~K}$ to $400 \mathrm{~K}$ (the upper limit of our measurement instrument, see Methods section) and different bias currents. The near perfect linearity of the Hall voltage as a function of magnetic field is seen to hardly depend on temperature. The current-related sensitivity $S_{\text {I }}$ as a function of temperature is shown in Fig. 4b. The sensitivity is seen to increase, from $494 \mathrm{~V} / \mathrm{AT}$ at $1.8 \mathrm{~K}$, with increasing temperature and peak at about $580 \mathrm{~V} / \mathrm{AT}$ at $350 \mathrm{~K}$, and then decreases with temperature. The temperature coefficient of the sensitivity is defined $\mathrm{as}^{26-28}$

$$
\gamma_{\mathrm{T}}=\frac{1}{S} \frac{\partial S}{\partial T}
$$

where $S$ denotes either the absolute sensitivity or the current/voltagerelated sensitivity. The temperature coefficient $\gamma_{\mathrm{T}}$ of the GHE operating in current mode remains below $800 \mathrm{ppm} \cdot \mathrm{K}^{-1}$ over a wide temperature range from $1.8 \mathrm{~K}$ to $400 \mathrm{~K}$ (Fig. 4c). As a comparison, Hall elements made from high mobility InSb usually has a much larger temperature coefficient of $-2000 \mathrm{ppm} \cdot \mathrm{K}^{-1}$ at $300 \mathrm{~K}^{4}$.

The output Hall voltage of the GHE is also measured as a function of the magnetic field in voltage mode at a bias of $2 \mathrm{~V}$ for various temperatures ranged from $1.8 \mathrm{~K}$ to $400 \mathrm{~K}$ (Fig. 4d). It is important to note that the $\mathrm{V}_{\mathrm{H}}-\mathrm{B}$ curves at different temperature almost coincide with each other, suggesting that the behavior of the GHE is extremely stable within the $\sim 400 \mathrm{~K}$ temperature range in voltage mode (Fig. 4e). The temperature coefficients of the GHE in voltage mode are found to be smaller than $700 \mathrm{ppm} \cdot \mathrm{K}^{-1}$ from $1.8 \mathrm{~K}$ to $400 \mathrm{~K}$ (Fig. 4f). In particular, the temperature coefficient is as low as $30 \mathrm{ppm} \cdot \mathrm{K}^{-1}$ below $200 \mathrm{~K}$, which is much better than that of all published conventional Hall elements with typical temperature coefficient of around $1000 \mathrm{ppm} \cdot \mathrm{K}^{-14,26-28}$. On the other hand, the offset voltage is also found to hardly change over a wide temperature range from $1.8 \mathrm{~K}$ to $400 \mathrm{~K}$, which fluctuates between $-4 \mathrm{mV}$ and $-5 \mathrm{mV}$ (see Fig. S6 of supplementary information). The equivalent magnetic induction offset field $\mathrm{B}_{\mathrm{o} \text {,eq }}$ (defined as $V_{\text {offset }} / S_{\mathrm{A}}$,) is then about $-2 \mathrm{mT}$, which is much smaller than that of Si Hall elements (usually larger than $10 \mathrm{mT}$ ) and comparable to that of InGaAs-based Hall element (typically $2 \mathrm{mT})^{3}$. Since $\mathrm{B}_{\mathrm{o} \text {,eq }}$ is inversely proportional to carrier mobility, further reduction in $\mathrm{B}_{\mathrm{o} \text {,eq }}$ to sub milli-tesla range can be expected for GHEs.

The excellent thermal stability of GHE is originated from the intrinsic properties of graphene. It is well known that the thermal stability of a Hall element is determined by the temperature dependence of carrier density in current mode and mobility in voltage mode. For a conventional semiconductor, intrinsic carrier concentration is dependent on temperature T via an exponential law, i.e. $n_{i} \sim \exp (\mathrm{AT})$ (A being a constant), while carrier concentration in graphene is dependent on temperature much more weakly via a power law ${ }^{29}$, i.e. $n_{i} \propto \mathrm{T}^{2}$. Therefore, in principle GHE will exhibit better thermal stability than conventional Hall element in current mode. The temperature dependent carrier density and mobility of a typical GHE are shown in Fig. 5a. At temperature range from $400 \mathrm{~K}$ to $1.8 \mathrm{~K}$, carrier density changes about $17 \%$ from about $1.27 \times 10^{12} / \mathrm{cm}^{2}$ to $1.07 \times 10^{12} /$ $\mathrm{cm}^{2}$. In contrast, carrier mobility shows hardly any change and remains as a constant value from $1.6 \mathrm{~K}$ to $300 \mathrm{~K}$, and changes less than $7 \%$ from $2700 \mathrm{~cm}^{2} / \mathrm{V} \cdot \mathrm{s}$ at $300 \mathrm{~K}$ to $2500 \mathrm{~cm}^{2} / \mathrm{V} \cdot \mathrm{s}$ at $400 \mathrm{~K}$. Since the carrier mobility in our GHE is below $3000 \mathrm{~cm}^{2} / \mathrm{V} \cdot \mathrm{s}$, the mobility is limited mainly by the Coulomb scattering mechanisms, which is almost independent of temperature ${ }^{30-32}$, suggesting excellent thermal stability of GHEs in voltage mode.

Excellent thermal stability over a wide temperature range from $1.8 \mathrm{~K}$ to $400 \mathrm{~K}$ is crucial for many applications of high performance Hall elements in such fields as aircraft, spacecraft, military, oil and gas industries ${ }^{1-3,26,28}$. In a conventional semiconductor, carrier density and mobility are both temperature sensitive and the thermal
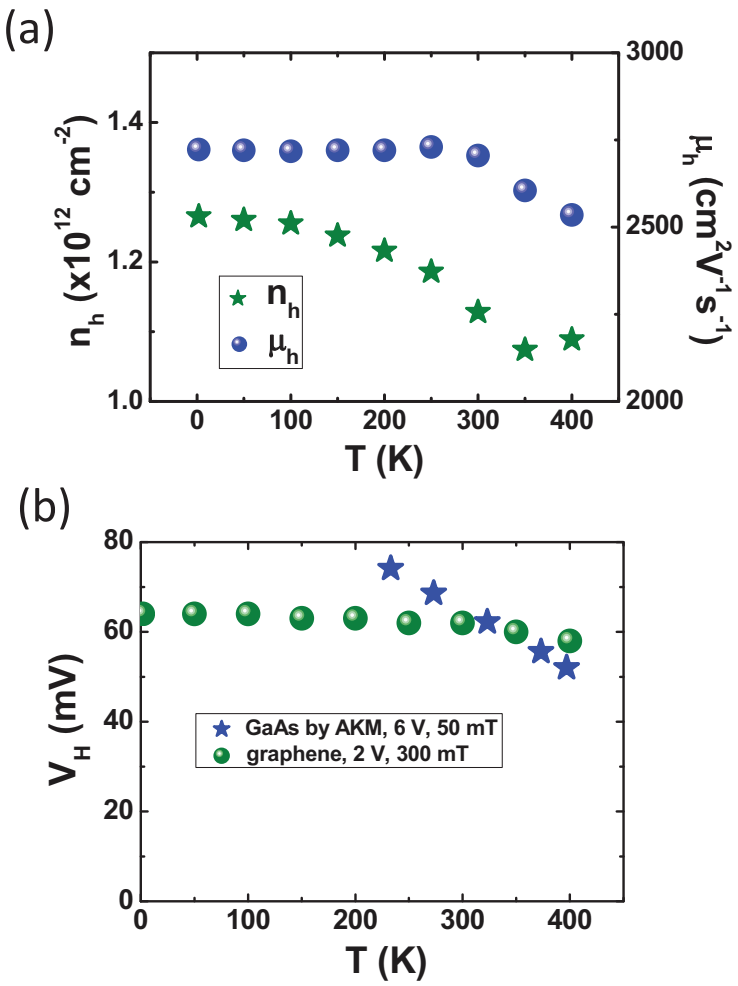

Figure 5 Temperature characteristics of graphene Hall elements and comparison with commercialized Hall elements supplied by Asahi Kasei Microdevices (AKM) Corporation. (a) Temperature dependent carrier density and Hall mobility of a typical GHE. Carrier mobility was retrieved under $\mathrm{B}=1 \mathrm{~T}$. (b) Temperature dependent Hall voltage of graphene (green spheres) and GaAs (blue stars) Hall elements in voltage mode. The voltage supplies are $2 \mathrm{~V}$ and $6 \mathrm{~V}$, and the magnetic fields are $300 \mathrm{mT}$ and $50 \mathrm{mT}$ for graphene and GaAs Hall elements respectively. The specification for the commercial AKM GaAs Hall element is HG-106C-2U.

stability of the corresponding Hall element is generally poor ${ }^{26-28}$. Low thermal drift is usually realized by heavily doping the semiconductor, but doping reduces mobility and increases carrier density leading to a lowered sensitivity of the Hall device. Compromise usually has to be made between low thermal drift and high sensitivity. In contrast, in graphene both carrier density and mobility depend only weakly on temperature ${ }^{30-32}$. The GHE thus exhibits a much better thermal stability than that of the conventional Hall device, and this is especially so when the GHE is used in voltage mode (Fig. 5b). In principle the GHE can have low carrier density and high mobility simultaneously over a wide temperature range, which represents a significant advantage over all commercial Hall devices as shown in Fig. S7 in supplementary information.

In summary, graphene is an ideal material for building high-performance general purpose Hall elements with low cost and in largescale. Compared with conventional Hall elements, the sensitivity of our GHE is as high as that of the bests based on 2DEG, and the sensitivity could potentially be further improved significantly by optimizing fabricating process. The combination of high sensitivity and low noise of graphene devices leads to ultra-high resolution, and in principle GHEs could be used for detecting extremely low magnetic field. The graphene Hall device also presents excellent linearity over a wide magnet field, especially in voltage mode. In addition, because in graphene both carrier density and mobility depend only weakly on temperature, GHE can operate linearly at ultra-wide temperature range at least from $1.8 \mathrm{~K}$ to $400 \mathrm{~K}$ simultaneously with high sensitivity and low thermal drift, extending the application field of GHE significantly. Last but not the least, methods for growing and 
transferring high quality graphene have been developed, providing possibility for large-scale fabrication of GHEs which take the full advantages of the excellent electric properties of graphene. In addition, the GHE fabrication process is low cost, compatible with that of signal-processing circuits, and may be carried out on different substrate, such as ultra-light material or flexible substrate. Graphene Hall devices are therefore general purpose high-performance magnetometers which can potentially satisfy most kinds of requirements for a wide range of applications, and potentially ideal substitutes for conventional Hall elements.

\section{Methods}

Graphene growth and transfer. Graphene was synthesized on a $50 \mu \mathrm{m}$ thick polycrystalline $\mathrm{Pt}$ (with purity of $99.95 \%$ ) film at $1070^{\circ} \mathrm{C}$. Firstly, $\mathrm{Pt}$ film was heated to $1070^{\circ} \mathrm{C}$ under a constant flow of $300 \mathrm{sccm} \mathrm{H}_{2}$ in a quartz tube in $50 \mathrm{mins}$ by a Lindberg/Blue furnace. Secondly, a mixture of $5 \mathrm{sccm} \mathrm{CH}_{4}$ and $700 \mathrm{sccm} \mathrm{H} \mathrm{H}_{2}$ was introduced into the quartz tube to growth graphene. The growth time was usually 15 mins, which we found is enough for graphene to fully covering the Pt metal surface. Finally, a fast cooling process was taken by simply pulling the quartz tube out till the sample was no longer in the heating region of the furnace. The synthesized graphene was transferred to the $\mathrm{SiO}_{2} / \mathrm{Si}$ substrate by the bubbling transfer method described by Gao et al. ${ }^{19}$. The thickness of the $\mathrm{SiO}_{2}$ layer is $285 \mathrm{~nm}$.

Device fabrication. The patterns of metal contacts and pads of the graphene Hall element were defined by electron beam lithography (EBL) on poly methyl methacrylate (PMMA). A $5 \mathrm{~nm} \mathrm{Ti/45} \mathrm{nm} \mathrm{Au} \mathrm{metal} \mathrm{stack} \mathrm{was} \mathrm{then} \mathrm{deposited} \mathrm{by}$ electron beam evaporation (EBE), followed by a lift-off process in acetone. The graphene was tailored to rectangle geometry by EBL on PMMA followed by developing and oxygen plasma etching.

Fabrication of Gauss meter. The graphene Gauss meter is mainly composed of three parts, i.e. a magnetic-field-sensitive probe, a power supply of the probe, and a voltmeter which is used to measure the Hall voltage generated by the probe. The key component of the probe is a macro graphene Hall element fabricated by the processes described in the main text. The chip carrying the graphene Hall element was placed on a printed circuit board (PCB), with all the four metal contacts connected to the electrical wires on the PCB board by thin gold threads with a diameter of $50 \mu \mathrm{m}$. In addition, small amount of indium was used to soldering gold threads with metal leads where necessary. The power supply was made by two batteries in series, with a total constant voltage output of 3.0 V. The voltmeter used is Agilent 34410A Digit Millimeter.

Surface modification of APTES. The surface modification using APTES was done in two steps. First, the $\mathrm{SiO}_{2} / \mathrm{Si}$ substrate was treated to be hydrophilic in piranha solution $\left(\mathrm{H}_{2} \mathrm{O}_{2}: \mathrm{H}_{2} \mathrm{SO}_{4}=1: 3\right)$ for 5 minutes at room temperature, rinsed with DI water and dried by nitrogen. Then, the substrate was transferred to the APTES solution (APTES: IPA $=1: 100$ ). After a reaction time of 10 minutes, the $\mathrm{SiO}_{2} / \mathrm{Si}$ substrate was taken out, rinsed with IPA and dried by nitrogen.

Estimating the resolution limit of GHE. The resolution of a Hall element can be written as $B_{\min }=N_{V} / S_{A}$, where $N_{V}$ is the voltage noise spectral density at the device output, and $S_{A}$ is the absolute sensitivity of the Hall element ${ }^{10}$. To be simple, we will only consider thermal noise, which is usually the dominant noise spectrum at high frequencies. Then, we have $N_{V} \approx \sqrt{4 k_{B} T R_{\text {out }}}$, in which $\mathrm{k}_{\mathrm{B}}$ is the Boltzmann constant, $\mathrm{T}$ is temperature and $R_{\text {out }}$ is the output resistance of the Hall element, i.e. the resistance measured between $\mathrm{C}_{3}$ and $\mathrm{C}_{4}$ as in figure $2(\mathrm{~b})$. The absolute sensitivity is estimated through $S_{A}=W \cdot v_{d}$, which is a result of the balance between the Hall electric field and the Lorentz force. Here $\mathrm{W}$ is the width of the Hall plate and $v_{d}$ is the drift velocity of the charge carriers. As a result, we have $B_{\min } \approx \sqrt{4 k_{B} T R_{\text {out }}} /\left(W \cdot v_{d}\right)$. It's clear that material with higher saturation drift velocity $v_{\text {sat }}$ has greater potential in building Hall elements with higher resolution. Notice that even the longitude of the Hall plate is stressed with high electric field and the velocity of the charge carriers tends to saturate, $R_{\text {out }}$ may be still estimated by the diffusive model with $R_{\text {out }} \approx(q n \mu)^{-1}$, where we suppose the effective width-to-length ratio related with $R_{\text {out }}$ is typically 1 . Thus resolution of GHE normalized by channel width can be given by $B_{\min } \cdot W \approx \sqrt{4 k_{B} T /\left(q n \mu v_{\text {sat }}^{2}\right)}$. It's very clear that both high mobility and high saturation velocity of carriers in graphene will contribute to decrease the minimum detectable magnetic field, or similarly improve the resolution. Using room temperature reference value in high-quality graphene, including $\mu=5 \mathrm{~m}^{2} / \mathrm{V} \cdot \mathrm{s}$, $v_{\text {sat }}=4 \times 10^{5} \mathrm{~m} / \mathrm{s}$, and density $=1 \times 10^{12} \mathrm{~cm}^{-2}$, we can obtain

$B_{\min } \cdot W \sim 4 \mathrm{pT} \cdot \mathrm{mm} / \sqrt{\mathrm{Hz}}$.

Temperature dependent Hall measurement. The device test was carried out in a modified MPMS (SQUID) VSM 7 Tesla System, with which a maximum 7independent-port electrical signal measurement was possible. The constant voltage or current source of the graphene hall device was supplied by a lock-in amplifier Li5640 by $\mathrm{nF}$ cooperation, which also measures the hall voltage generated by the hall device but at different aux ports. The resolution of the hall voltage measurement is $1 \mathrm{mV}$.
The back gate voltage was supplied by Keithley 2400 Source Meter. A 30 minutes in situ annealing in the vacuum chamber of the MPMS system at $400 \mathrm{~K}$ was carried out before any electrical measurement.

1. Boero, G., Demierre, M., Besse, P. A. \& Popovic, R. S. Micro-Hall devices: performance, technologies and applications. Sensors and Actuators A 106, 314-320 (2003).

2. Ripka, P. \& Janosek, M. Advances in Magnetic Field Sensors. IEEE Sensors Journal 10, 1108-1116 (2010).

3. Popovic, P. S., Flanagan, J. A. \& Besse, P. A. The Future of magnetic Sensors. Sensor and Actuators A 56, 39-55 (1996).

4. Mihajlovic, G., Xiong, P., von Molnar, S., Ohtani, K., Ohno, H., Field, M. \& Sullivan, G. J. Detection of single magnetic bead for biological applications using an InAs quantum-well micro-Hall sensor. Appl. Phys. Lett., 87, 112502 (2005).

5. Popovic, R. S. Hall Effect Devices, Second Edition, Institute Physics Publishing, 2004.

6. Heremans J. Solid State Magnetic Field Sensors and Applications. J. Phys. D: Appl. Phys. 26, 1149-1168 (1993).

7. Hara, T., Mihara, M., Toyoda, N. \& Zama, M. Highly Linear GaAs Hall Devices Fabricated by Ion Implantation. IEEE Transactions on Electron Devices ED-29, 78-82 (1982).

8. Shibasaki, I. Mass production of InAs Hall elements by MBE. Journal of Crystal Growth 175/176, 13-21(1997).

9. Berus, T., Oszwaldowski, M. \& Grabowski, J. High quality Hall sensors made of heavily doped n-InSb epitaxial films. Sensors and Actuators A1 16, 75-78 (2004).

10. Kunets, V. P., Black, W. T., Mazur, Y. I., Guzun, D., Salamo, G. J., Goel, N., Mishima, T. D., Deen, D. A., Murphy, S. Q. \& Santos, M. B. Highly Sensitive Micro-Hall Devices Based on $\mathrm{Al}_{0.12} \mathrm{In}_{0.88} \mathrm{Sb} / \mathrm{InSb}$ Heterostructures. J. Appl. Phys. 98, 014506 (2005).

11. Bando, M., Ohashi, T., Dede, M., Akram, R., Oral, A., Park, S. Y., Shibasaki, I., Handa, H. \& Sandhu, A. High sensitivity and multifunctional micro-Hall sensors fabricated using InAlSb/InAsSb/InAlSb heterostructures. J. Appl. Phys. 105, 073909 (2009).

12. Schwierz, F. Graphene Transistor. Nature Nanotechnol 5, 487-496 (2010).

13. Lin, Y. M., Valdes-Garcia, A., Han, S. J., Farmer, D. B., Meric, I., Sun, Y. N., Wu, Y. Q., Dimitrakopoulos, C., Grill, A., Avouris, P. \& Jenkins, Keith A. Wafer-Scale Graphene Integrated Circuit. Science 332, 1294-1297 (2011).

14. Schedin, F., Geim, A. K., Morozov, S. V., Hill, E. W., Blake, P., Katsnelson, M. I. \& Novoselov, K. S. Detection of individual gas molecules adsorbed on graphene. Nat. Mater. 6, 652-655 (2007).

15. Mueller, T., Xia, F. \& Avouris, P. Graphene photodetectors for high-speed optical communications. Nature Photonics 4, 297-301 (2010).

16. Novoselov, K. S., Jiang, Z., Zhang, Y., Morozov, S. V., Stormer, H. L., Zeitler, U., Maan, J. C., Boebinger, G. S., Kim, P. \& Geim, A. K. Room-Temperature Quantum Hall Effect in Graphene. Science 315, 1379 (2007).

17. Pisana, S., Braganca, P. M., Marinero, E. E. \& Gurney, B. A. Tunable Nanoscale Graphene Magnetometers. Nano Lett 10, 341-346 (2010).

18. Lu, J. M., Zhang, H. J., Shi, W., Wang, Z., Zheng, Y., Zhang, T., Wang, N., Tang, Z. K. \& Sheng, P. Graphene Magnetoresistance Device in van der Pauw Geometry. Nano Lett 11, 2973-2977 (2011).

19. Tang, C. C., Li, M. Y., Li, L. J., Chi, C. C. \& Chen, J. C. Characteristics of a sensitive micro-Hall probe fabricated on chemical vapor deposited graphene over the temperature range from liquid-helium to room temperature. Applied Physics Letters 99, 112107 (2011).

20. Panchal, V., Cedergren, K., Yakimova, R., Tzalenchuk, A., Kubatkin, S. \& Kazakova, O. Small epitaxial graphene devices for magnetosensing applications. Journal of Applied Physics 111, 07E509 (2012).

21. Gao, L. B., Ren, W. C., Xu, H. L., Jin, L., Wang, Z. X., Ma, T., Ma, L. P., Zhang, Z. Y., Fu, Q., Peng, L. M., Bao, X. H. \& Cheng, H. M. Repeated growth and bubbling transfer of graphene with millimetre-size single-crystal grains using platinum. Nature Comm 3, 3,699 (2012).

22. Park, J., Jo, S. B., Yu, Y. J., Kim, Y., Yang, W., Lee, W. H., Kim, H. H., Hong, B. H., Kim, P., Cho, K. \& Kim, Kwang S. Single-Gate Bandgap Opening of Bilayer Graphene by Dual Molecular Doping. Adv. Mater. 24, 407-411 (2012).

23. Moon, J. S., Curtis, D., Zehnder, D., Kim, S., Gaskill, D. K., Jernigan, G. G., MyersWard, R. L., Eddy, C. R., Campbell, P. M., Lee, K.-M. \& Asbeck, P. Low-phasenoise Graphene FETs in Ambipolar RF applications. IEEE Electron Device Letters 32, 270-272 (2011).

24. Du, X., Skachko, I., Barker, A. \& Andrei, E. Y. Approaching ballistic transport in suspended graphene. Nature Nanotechnol 3, 491-495 (2008).

25. Dean, C. R., Young, A. F., Meric, I., Lee, C., Wang, L., Sorgenfrei, S., Watanabe, K., Taniguchi, T., Kim, P., Shepard, K. L. \& Hone, J. Boron nitride substrates for highquality graphene electronics. Nature Nanotechnol 5, 722-726 (2010).

26. Jankowski, J., El-Ahmar, S. \& Oszwaldowski, M. Hall Sensors for Extreme Temperatures. Sensors 11, 876-885 (2011).

27. Kunets, V. P., Dobbert, J., Mazur, Y. I., Salamo, G. J., Muller, U., Masselink, W. T., Kostial, H. \& Wiebicke, E. Low Thermal Drift in Highly Sensitive doped channel $\mathrm{Al}_{0.3} \mathrm{Ga}_{0.7} \mathrm{As} / \mathrm{GaAs} / \mathrm{In}_{0.2} \mathrm{Ga}_{0.8} \mathrm{As}$ Micro-Hall Element. Journal of Materials Science: Materials in Electronics 19, 776-782 (2008). 
28. Lu, H., Sandvik, P., Vertiatchikh, A., Tucker, J. \& Elasser, A. High temperature Hall effect sensors based on AlGaN/GaN heterojunctions. J. Appl. Phys. 99, 114510 (2006)

29. Fang, T., Konar, A., Xing, H. L. \& Jena, D. Carrier statistics and quantum capacitance of graphene sheets and ribbons. Applied Physics Letters 91, 092109 (2007).

30. Adam, S., Hwang, E. H., Galitski, V. M. \& Das Sarma, S. A Self-consistent Theory for Graphene Transport. Proc. Nat. Acad. Sci. USA 104, 18392-18397 (2007).

31. Chen, J.-H., Jang, C., Adam, S., Fuhrer, M. S., Williams, E. D. \& Ishigami, M. Charged-Impurity Scattering in Graphene. Nat. Phys. 4, 377-381 (2008).

32. Zhu, W. J., Perebeinos, V., Freitag, M. \& Avouris, P. Mobilities and electrostatic potential in monolayer, bilayer, and trilayer graphene. Phys. Rev. B 80, 235402 (2009).

\section{Acknowledgements}

This work was supported by the Ministry of Science and Technology of China (Grant Nos. 2011CB933001 and 2011CB933002), and Special Financial Grant from the China

Postdoctoral Science Foundation (Grant No. 2012T50020). The authors would like to thank
Prof. L. Lv and Dr. F. Yang for providing laboratory facilities for some of the electrical measurements reported in this work.

\section{Author contributions}

Z.Z. and L.M.P. designed the experiment, H.X. and R.S. performed the growth and transfer of graphene, device fabrication and characterization. H.X. and Z.W. performed the noise measurement of GHE. H.X., Z.Z. and L.M.P. analysed the data and co-wrote the manuscript. All authors discussed the results and commented on the manuscript.

\section{Additional information}

Supplementary information accompanies this paper at http://www.nature.com/ scientificreports

Competing financial interests: The authors declare no competing financial interests. License: This work is licensed under a Creative Commons

Attribution-NonCommercial-NoDerivs 3.0 Unported License. To view a copy of this license, visit http://creativecommons.org/licenses/by-nc-nd/3.0/

How to cite this article: HuilongXu et al. Batch-fabricated high-performance graphene Hall elements. Sci. Rep. 3, 1207; DOI:10.1038/srep01207 (2013). 